\title{
La Organización Internacional del Trabajo y el problema social indígena: La encuesta en Perú de 1936
}

\section{The International Labour Organization and the Indigenous Social Problem: The 1936 Survey on Peru}

\author{
Juan Carlos Yáñez Andrade \\ Facultad de Ciencias Económicas y Administrativas \\ Universidad de Valparaíso, Chile \\ ORCID: 0000-0003-0317-3292 \\ juancarlos.yanez@uv.cl
}

Resumen: Este artículo aborda la encuesta realizada en Perú en 1936 por la Organización Internacional del Trabajo con el fin de conocer el problema social indígena. A partir de las resoluciones de la Conferencia Interamericana del Trabajo de 1936 y el informe elaborado por Moisés Poblete Troncoso se muestra la importancia que tienen los nuevos problemas sociales en las instancias de cooperación técnica del periodo. La encuesta de Moisés Poblete no sólo ayudará a conocer mejor la realidad indígena peruana, sino que también servirá de insumo para las acciones futuras de la Organización Internacional del Trabajo en la materia, proceso que finalizará, en una primera etapa, con la convención 107 de 1957.

Palabras clave: OIт; Moisés Poblete; problema social indígena; encuesta; Perú; Conferencia Americana del Trabajo.

Abstract: This article discusses the survey conducted in Peru in 1936 by the International Labour Organization to understand the indigenous social problem. The resolutions of the 1936 Inter-American Labour Conference 
and the report by Moisés Poblete Troncoso revealed the importance of the new social problems for the technical cooperation organizations of the time. Moisés Poblete's survey not only contributed to an understanding of the Peruvian indigenous reality, but also served as input for future actions by the International Labour Organization in the matter, the first stage of which was completed at the 107th Convention in 1957.

Key words: ILO; Moisés Poblete; indigenous social problem; survey; Peru; American Labor Conference.

Fecha de recepción: 29 de enero de 2016 Fecha de aceptación: 3 de octubre de 2016

\section{INTRODUCCIÓN}

$\mathrm{L}$ os años 1920 y 1930 fueron clave en el internacionalismo latinoamericano. Si desde fines del siglo xix las conferencias panamericanas se transformaron en espacios propicios para el contacto diplomático, ${ }^{1}$ facilitando el desarrollo de conferencias científicas y congresos internacionales, fue con la creación de la Sociedad de las Naciones (SDN) en 1919 que se promovieron aún más las relaciones internacionales y los vínculos de cooperación continental (Dumont, 2008; Wehrli, 2003).

En nuestro estudio adquiere relevancia la Organización Internacional del Trabajo (ОIт), parte integrante del complejo de instituciones de la SDN y encargada de promover el mejoramiento de las condiciones laborales en el mundo. ${ }^{2}$ El Consejo de Administración (CA), órgano ejecutivo de la oIT y elegido por la conferencia, designó a Albert Thomas como su primer director, el cual permanecería en el cargo hasta su muerte en 1932. El primer año de existencia de la ort fue de plena actividad, teniendo como principal prioridad el darse una constitución formal y resolver sobre las disposiciones más urgentes en materia laboral. La primera reunión de la Conferencia

${ }^{1}$ No hay una verdadera bibliografía consagrada al panamericanismo, a excepción de algunos textos de comienzos del siglo xx. Véase Gallardo (1941); Kelchner (1931); Manger (1931); Naón (1919); Quesada (1916, 1919). Recientemente se ha publicado el libro de Sheinin (2000) y uno sobre la participación de México en las conferencias panamericanas (Marichal, 2002).

${ }^{2}$ En los últimos años se ha producido una revolución en los estudios de la oIt a partir de los enfoques transnacionales y comparados. Véase Aglan, Feiertag y Kévonian (2011); Kott y Droux (2012); Lespinet-Moret y Viet (2011); Van Daele (2010).

\section{()(1) $\$$}


Internacional del Trabajo se celebró a partir del 29 de octubre de 1919 en Washington y cada uno de los Estados miembros envió dos representantes gubernamentales, uno patronal y otro de los trabajadores, tal como lo establecían los reglamentos.

El sistema de aprobación de la legislación social internacional funciona a partir de un cuadro normativo complejo que comprende tres instancias. En primer lugar, la simple "recomendación", entendida como un voto de buena voluntad que los países son invitados a respectar. En segundo lugar, la "convención", comprendida como un acuerdo de deliberación de cada conferencia y que no tiene el carácter de ley hasta que no son ratificadas. En esta tercera instancia (la "ratificación"), los países se comprometen a someter las convenciones a sus parlamentos, para que sean estos los que se pronuncien sobre su incorporación en la legislación nacional. Sólo a partir de este momento se puede hablar de una verdadera legislación social internacional.

Los años de la década de 1930 se iniciaron con la Gran Depresión, que en América Latina golpeó principalmente al comercio exterior, afectando la exportación de materias primas, clave en el flujo de capitales. Esto se tradujo en reducción presupuestaria y contracción del mercado interno, con las consecuencias en la pérdida del poder de compra de la población, el aumento de la cesantía y una recesión económica que duraría años (Drinot y Knight, 2015). En este marco los países latinoamericanos se vieron confrontados a problemas nuevos, que requerían transformaciones estructurales y una cooperación a nivel regional como nunca había sido planteada, mostrándose las organizaciones del sistema panamericano y de la SDN dispuestas a promover programas de asistencia técnica, como forma de apoyar el esfuerzo desplegado por los gobiernos en mejorar su legislación laboral y seguridad social (Plata, 2013; Yáñez, 2014).

La OIT se interesó en estos años por acercarse a los países latinoamericanos, más allá del poco compromiso manifestado por estos en ratificar las convenciones internacionales del trabajo. Para la organización de Ginebra, América Latina era un aliado clave en su propio proceso de consolidación institucional, cuando la participación de Asia y África era prácticamente inexistente y Europa se hallaba bajo el peligro del avance del nazismo. Este interés de la оіт por la región suponía estar abierta a sus demandas y ofrecer nuevas instancias de acercamiento que fueran más allá de las conferencias internacionales y su enfoque universalista. Esto fue el origen de las conferencias interamericanas convocadas por la OIт y desarrolladas en distintos países latinoamericanos entre 1936 y 1949, las que por su carácter regional

\section{()(1) $(3$}


y su representación tripartita (con delegados gubernamentales, sindicales y empresariales) se transformaron en espacios de debate sobre las particularidades del continente. ${ }^{3}$ Entre estas particularidades la situación de los indígenas apareció en los años de la década de 1930 no sólo como algo distintivo para América Latina, sino también como una de las vías que tuvo la oit para integrar en su ámbito de acción a los países andinos, mismo papel que tuvo para el resto de los países el trabajo marítimo, el agrícola o la alimentación popular (Caruso, 2016; Pernet, 2011). En este sentido reconocemos que la problemática indígena era un aspecto de interés para países de otras latitudes, como la India, los países balcánicos, Turquía u otros, pero en nuestra perspectiva esta problemática ofreció la ventaja de insertarse en un cambio de estrategia de la ort en su vinculación con el continente, pasando de un interés por la ratificación de las convenciones internacionales del trabajo a uno que lo complementaba con asesorías y asistencia técnica. ${ }^{4}$ Parte de esta asistencia técnica comprendía la realización de encuestas, la capacitación del personal administrativo o la redacción de proyectos legislativos, lo que suponía reconocer ciertas debilidades de las burocracias nacionales en implementar políticas y programas de acción social. Sin embargo, como tendremos ocasión de verlo a propósito de la encuesta que llevó a cabo la ort en Perú, estaba lejos de resolverse el dilema de cómo pensar los problemas sociolaborales del continente desde la matriz teórica de la ort, donde el obrero era entendido en su dimensión industrial e ideológica (organizado en torno a un movimiento obrero), muy lejos de la realidad mayoritaria que comprendía la población campesina, artesanal e indígena.

Es necesario, en todo caso, señalar que la condición de los indígenas se había transformado en un tema de preocupación para la oIT mucho antes de los años treinta del siglo pasado, proponiendo en 1921 el desarrollo de estudios sobre la condición laboral de este grupo y creando en 1926 la Comisión de Expertos en Trabajo Nativo (González, 2000). A partir de los años de la década de 1930, y con el giro latinoamericano que estaba teniendo la oIT, la realidad indígena adquirió una dimensión nueva, prueba de la apertura a uno de los temas más sensibles de la región (Rodríguez-Piñero, 2005). Este acercamiento se vio apoyado por un cuadro creciente de funcionarios y re-

${ }^{3}$ En orden cronológico fueron las siguientes. En 1936 se desarrolló en Santiago de Chile la Primera Conferencia Americana del Trabajo; la segunda en La Habana en 1939; la tercera en la ciudad de México en 1946, y la cuarta se desarrolló en Montevideo en 1949.

${ }^{4}$ Para un programa de asistencia técnica en América del Sur durante los años 1930, véase Plata (2013).

\section{()(1) $(9$}


des con vocación continental, lo que supuso una temprana incorporación de sudamericanos a los órganos administrativos de la institución de Ginebra y corresponsalías establecidas en algunas capitales como Buenos Aires y Santiago de Chile. En este sentido, funcionarios como Moisés Poblete, Carlos García Palacios, Raúl Migone, entre otros, fueron representativos de este universo latinoamericano de la oIT.

El objetivo central de este artículo es analizar la encuesta realizada en Perú en 1936 por Moisés Poblete Troncoso, funcionario de la oit, en el marco de una de las resoluciones aprobadas por la Conferencia Americana del Trabajo (САT) realizada en Santiago de Chile en enero de ese año. Tal resolución sugería, en primer lugar, la necesidad de que los países latinoamericanos con mayor población indígena entregaran la información necesaria para conocer los problemas sociales, económicos y laborales de dicha población, y, en segundo lugar, que la oiт iniciara un estudio sobre la materia con el fin de proponer acciones a nivel internacional (Organización Internacional del Trabajo [ort], 1936, pp. 147-148). Si bien su director, Harold Butler, encomendó la realización de una encuesta en Perú, Bolivia, Ecuador y Colombia, Moisés Poblete pudo llevar a cabo su cometido sólo en el primer país.

Proponemos como hipótesis que el interés que mostraron las autoridades de algunos países, así como los funcionarios de la oit por conocer el problema social indígena, se vio enfrentado a la falta de experiencias previas, la propia evolución que estaba teniendo la institución de Ginebra al pasar de un foco colocado en la ratificación de las convenciones del trabajo a uno de cooperación técnica, la ausencia de estadísticas confiables y las distintas formas de entender el problema indígena por parte de las autoridades locales e intelectuales, lo que puede explicar el desconocimiento de este trabajo, apareciendo como un intento aislado por conocer la situación de los indígenas. ${ }^{5}$

$\mathrm{El}$ artículo lo hemos estructurado en tres secciones que comprenden, primero, una presentación de las condiciones que hicieron posible la convocatoria a la primera CAT realizada en Santiago de Chile en 1936 y que dentro de sus resoluciones estuvo el encargar la realización de una encuesta sobre la condición indígena en algunos países de la costa del Pacífico sudamericano. Luego se presentan los principales problemas que relevó la conferencia de

${ }^{5}$ No deja de ser demostrativo del desconocimiento del informe de Poblete el que un historiador que busca ofrecer una perspectiva "racial" de la constitución de las políticas sociales en el Perú de los años 1930 y 1940, a través del estudio del proyecto de "desindianización” de la nación peruana, como es Drinot (2016), ni siquiera cite la encuesta de 1936.

\section{()(1) $\$$}


Santiago de Chile, destacando la cuestión indígena como un resorte temático que permitió el acercamiento de la OIт a los problemas sociales del continente, para finalizar con la encuesta del funcionario de la oIT, Moisés Poblete, realizada en Perú en 1936.

Para cumplir con el objetivo de este artículo hemos utilizado las fuentes disponibles en el archivo de la ort en Ginebra, en el Archivo Nacional de Chile, además de las actas de la CAT y el informe elaborado por Moisés Poblete en Perú y publicado en 1938. El conjunto de esta documentación nos permitirá dimensionar la importancia de esta encuesta en hacer visible el problema social indígena y las instancias trasnacionales que se articulan para abordar esta realidad presente en muchos países.

\section{LA CONFERENCIA AMERICANA DEL TRABAJO DE 1936}

La CAT convocada por la oIT y desarrollada en Santiago de Chile en 1936 supuso un espacio privilegiado para discutir sobre los problemas sociales del continente (Herrera, 2013b). El delegado gubernamental chileno, Fernando García Oldini (1935), cuando invitó a los delegados de la XIX Conferencia Internacional del Trabajo a celebrar una conferencia regional, justificó tal reunión "para discutir no sólo las cuestiones de importancia universal, sino que también cuestiones regionales, como las condiciones de los trabajadores agrícolas, la utilización del tiempo libre de los trabajadores y la aplicación de la seguridad social" (p. 426). Dicha propuesta generó la adhesión inmediata de todos los representantes americanos presentes en Ginebra, aprobándose el 21 de junio de 1935 en la sesión número 72 del Consejo de Administración de la orT.

El esquema tripartito de las conferencias internacionales siempre fue destacado como lo más novedoso y progresista de la OIT, al incorporar en sus debates a los representantes gubernamentales, empresariales y sindicales en la resolución y aprobación de las convenciones. Reglamentariamente la organización de la CAT a realizarse en la ciudad de Santiago debía estar a cargo del CA, siendo financiada con aportes de la institución de Ginebra y del gobierno de Chile. ${ }^{6}$ El cA preparó el reglamento y cinco informes sobre

${ }^{6}$ Carta confidencial del delegado chileno en Ginebra, García Oldini. 11 de julio y 5 de agosto de 1935. Ministerio del Trabajo. Oficios y providencias. Vol. 231, 1935. Archivo Nacional (en adelante AN), Chile.

\section{()(1) $\$$}


los temas a tratar en el programa. La propuesta mexicana de incorporar en el debate las condiciones laborales en la agricultura planteó serias dificultades a la representación brasileña, la que amenazó con marginarse del encuentro si se trataba dicho tema. México estaba en pleno proceso de reforma agraria y de sindicalización campesina, y deseaba dar a conocer su experiencia, lo que era considerado por Brasil como un asunto nacional, difícil de discutir en una conferencia de carácter continental. Sin embargo, el cA resolvió apoyar la proposición mexicana e incorporar en el programa de la CAT la situación agrícola del continente, argumentando que una materia tan fundamental no podía estar ausente de un encuentro que abordara temáticas sociales. ${ }^{7}$

Para organizar la participación de cada una de las delegaciones, los países constituyeron una comisión especial encargada de preparar la nómina de delegados y de los consejeros técnicos no gubernamentales, así como elaborar informes y proyectos de resolución. Se buscaba evitar los conflictos al interior de las delegaciones y un posible rechazo de las representaciones por sector, especialmente obreras. La comisión chilena estuvo presidida por el profesor universitario Héctor Escribar, experto en derecho laboral, ${ }^{8} \mathrm{y}$ asesorada por Carlos Dragoni, que investigaba en Chile, por mandato de la SDN, el problema de la alimentación, y Alejandro Dusseillant, responsable de estudiar las condiciones sociales de los trabajadores agrícolas. ${ }^{9}$

La CAT fue inaugurada el 2 de enero de 1936 en el salón de ceremonias del Congreso Nacional de Chile. De las 19 naciones presentes (Costa Rica envió un observador gubernamental), sólo diez enviaron delegaciones completas, permitiendo el encuentro de expertos en materia laboral y social como Afonso Bandeira de Mello, director general del Trabajo de Brasil y antiguo delegado gubernamental a las conferencias de la oIT; Exequiel González Cortés, antiguo diputado, médico y redactor de la ley de seguros sociales de Chile; Santiago Labarca, presidente de la Caja del Seguro Obrero de Chile; Edgardo Rebagliati, encargado en la época de la redacción del proyecto de seguro obrero obligatorio de Perú, y Alejandro Unsain, experto en derecho laboral y delegado argentino a las conferencias de la огт. La representación

${ }^{7}$ Carta confidencial del delegado chileno en Ginebra, García Oldini. 8 de agosto de 1935. Ministerio del Trabajo. Vol. 231. AN, Chile.

${ }^{8}$ Memorándum de la comisión chilena, sin fecha. Ministerio del Trabajo. Providencias. Vol. 278, 1936. AN, Chile.

${ }^{9}$ Comunicación del Ministerio del Trabajo al Ministerio de Relaciones Exteriores, 8 y 29 de octubre de 1935. Ministerio de Relaciones Exteriores. Oficios recibidos, Ministerios V. Vol. 3506, 1935. AN, Chile.

\section{()(1) $\$$}


de la oIT estuvo también garantizada con la presencia del experto David Blelloch, encargado de la ratificación y aplicación de las convenciones; W. Jenks, de los aspectos jurídicos; E. Siewers, de la cesantía, y Oswald Stein de los seguros sociales. El experto Maurice Stack formó parte de la comisión de seguros sociales y Adolf Staal fue nombrado responsable de las relaciones con los delegados obreros presentes en la conferencia. Por último, los funcionarios de la ort, el argentino S. L. Childs y el chileno Moisés Poblete Troncoso, fueron nombrados asistentes del secretario general de la CAT, cargo asumido por Harold Butler.

La CAT conformó tres comisiones, cada una constituida por delegados gubernamentales, sindicales y patronales, además de secretarios y un representante de la огт: la comisión de proposiciones, la de seguros sociales y la de trabajo infantil y de mujeres. En cuanto a los aspectos que podían ser objeto de debates, algunas delegaciones formularon las siguientes propuestas: Estados Unidos solicitó que se discutiera sobre la edad mínima para ingresar al mercado laboral, la racionalización de la industria textil y la reducción de la jornada en esta rama industrial. Chile propuso debatir sobre la alimentación popular, la inspección laboral, el salario mínimo y las condiciones de vida de los obreros agrícolas. ${ }^{10}$

Los discursos de inauguración expresaron el deseo de que la CAT favoreciera el acercamiento de la OIT a los problemas específicos del continente, así como el reforzamiento de su acción en el mundo. ${ }^{11}$ Durante la discusión plenaria algunos delegados plantearon el desafío de cómo resolver, desde un punto de vista colectivo, los problemas sociales que afectaban al conjunto de los países, tomando en cuenta que en Europa la cooperación se apoyaba en el desarrollo industrial más o menos común, lo que no ocurría en América Latina, donde la cooperación se hacía difícil por las diferencias sociales y económicas. Es más, muchos gobiernos justificaban el retraso en la legislación social, argumentando las particularidades de su realidad social, política y económica, levantando algunas veces la bandera del nacionalismo con el fin de impedir una acción regional conjunta.

${ }^{10}$ Comunicación del Ministerio del Trabajo al Ministerio de Relaciones Exteriores, 10 de diciembre de 1935. Ministerio de Relaciones Exteriores. Oficios recibidos. Ministerios, V. Vol. 3506, 1935. AN, Chile.

${ }^{11}$ Los discursos de apertura fueron pronunciados, entre otros, por el ministro de Relaciones Exteriores de Chile, Cruchaga Tocornal, por el presidente del Consejo de Administración de la OIT, señor Ridell, y por los representantes gubernamentales, patronales y sindicales del CA (oIT, 1936, pp. 3-15).

\section{()(1) $(3$}


Por su tradición internacionalista las delegaciones sindicales se mostraban más dispuestas a colaborar con las instancias de cooperación continental e incluso tenían una posición crítica frente a aquellos sectores que buscaban limitar los derechos sociales a las fronteras de cada país. El delegado sindical chileno, Luis Solís, señaló al respecto:

En primer lugar, refutamos que se exagere, con criterio anacrónico, la ponderación de los factores geográficos e históricos en la elaboración del Derecho. Por encima de ellos se ha desarrollado, sobre todo en forma vertiginosa en las últimas décadas, la superestructura técnica del capitalismo, que borra las diferencias, acorta las distancias y acentúa la interdependencia de los pueblos en el plano mundial (oIт, 1936, p. 67).

Era claro que, con la influencia creciente de la ort y la necesidad de pensar regionalmente los problemas sociales, las naciones latinoamericanas debían integrar la variable internacional al momento de pensar sus legislaciones nacionales, lo que suponía ser receptivo a las ideas sociales provenientes del extranjero y estar dispuesto a promover una base común en la legislación laboral, como signo de pertenencia al continente. Como lo señaló el delegado patronal peruano, Ramírez Otárola (оIт, 1936), la CAT debía proponerse como objetivo central el beneficiar a todos los trabajadores de las ventajas de una legislación protectora (p. 143). En el mismo sentido, el delegado patronal de Bolivia (oIT, 1936) propuso un proyecto de resolución sobre la necesidad de que los países se esforzaran en uniformar su legislación social a partir de los principios aprobados en las distintas convenciones (p. 176).

Sin embargo, para algunos delegados gubernamentales la cooperación debía limitarse a la realización de estudios y encuestas a nivel regional, además de comparar las tasas de ratificación de las convenciones, pero sin avanzar en la discusión, por ejemplo, de un derecho social americano. El hecho de que la CAT no tuviera un carácter legislativo y no pudiera aprobar convenciones, limitaba sus alcances a una discusión sobre el nivel alcanzado por los países y a la propuesta de resoluciones que serían reflejo de las necesidades puntuales de los gobiernos, pero donde la perspectiva continental quedaba ausente. Por temor a extender los debates, algunos delegados llamaron la atención sobre el énfasis excesivo que se colocaba en la mirada puramente legislativa de la cooperación, sin integrar la reforma económica o política, como base de un mejoramiento efectivo de las condiciones de la clase obrera. Por ejemplo, el delegado gubernamental de Haití, J. G. Silva (oIT, 1936), señaló:

\section{()(1) $(9$}


"Me parece una cosa vana cuánto esfuerzo realicen las naciones por desarrollar lo más ampliamente posible su legislación social, si se dejan en pie hechos económicos y políticos que tiendan a contrarrestar los efectos verdaderos a que está destinada la legislación social” (p. 81). Esta posición del delegado haitiano reflejaba tanto las posturas de aquellos que criticaban el interés excesivo que colocaba la ort en el programa de ratificación de las convenciones, como de aquellos que cuestionaban la legislación social universal porque no contemplaba reformas más profundas en el ámbito económico y social.

\section{LOS PROBLEMAS SOCIALES MÁS URGENTES EN 1936}

La CAT tuvo en sus primeras sesiones plenarias un largo debate donde se expresaron las posiciones de las delegaciones sobre el primer tema propuesto en la tabla de discusión: la ratificación y aplicación de las convenciones internacionales del trabajo. Los delegados resolvieron sobre la necesidad de que la oIT prosiguiera y aumentara su acción de difusión sobre las ventajas de la legislación laboral y las publicaciones sobre el estado social de los países del continente. $^{12}$

Otro aspecto fundamental que se debatió fue sobre los organismos de inspección laboral, que aparecía en todos los debates a propósito de la aplicación de las convenciones de la OIT. Numerosas dificultades en la aplicación de una legislación social internacional aparecían así ligadas a la ausencia en el continente de una cultura de inspección y de respecto a la legislación. ${ }^{13} \mathrm{La}$ oIt jamás abordó directamente el tema de los organismos técnicos a través de una convención. Si bien en 1919 se aprobó una recomendación general sobre la materia, fue en 1923 que se trató de manera más profunda con la reco-

12 En un informe de 1939 sobre la situación del continente, la OIT reiteró su posición crítica. A pesar del avance de 1933 y 1934 en el proceso de ratificación de las convenciones, América Latina había ratificado sólo 160 de los 661 acuerdos aprobados por la asamblea de los Estados miembros de la oit, es decir $24.2 \%$. En relación con la aplicación de las convenciones, el mismo informe señalaba que sólo algunos países entregaban regularmente información sobre el tema (oIт, 1939, p. 162).

13 Tres países sudamericanos habían intentado crear organismos de inspección del trabajo antes de la creación de la oIт: Argentina (1907), Chile (1907) y Uruguay (1913). La mayoría de las oficinas o departamentos del trabajo comenzaron como organismos de estadística, para asumir luego funciones de inspección. Al respecto, véase Lobato (2007); Soprano (2007); Yáñez (2008).

\section{()(1) $\$$}


mendación número 20, la que señaló la necesidad de avanzar en la creación de organismos de inspección laboral, con el objetivo de garantizar la aplicación de las convenciones y, en general, de todas las medidas de mejoramiento de las condiciones de los trabajadores. De esta forma los gobiernos tuvieron libertad para ir estableciendo instituciones y procedimientos de inspección a medida que la legislación social avanzaba, lo que redundó en la instauración de un sistema poco uniforme a nivel continental, dotado de medios limitados y con dificultades en el reclutamiento de personal calificado. Por otra parte, la complejidad creciente de la legislación social ejerció una presión extra sobre la inspección, generando dudas sobre su autonomía real de los intereses empresariales. ${ }^{14}$

La CAT resolvió perfeccionar los procedimientos de inspección laboral, incluyendo una sección masculina y otra femenina, dotadas de las mismas facultades y derechos (Ministerio de Industria y Trabajo, 1937, pp. 29-30). La OIT no quedó al margen de esta evolución institucional y creó una sección denominada "Derecho e inspección del trabajo", responsable de realizar las tareas preparatorias de un estudio internacional sobre los principios generales que debía contener la organización de una inspección laboral moderna.

Un tercer tema sensible para los delegados a la cat fue el relativo a la agricultura, siendo los representantes mexicanos quienes monopolizaron el debate. La delegación mexicana presentó un informe sobre la situación en su país mostrándose como un modelo para el resto de las naciones del continente. El informe mostraba el progreso en materia de contrato de trabajo agrícola, comprendido tanto en la Constitución de la república como en la Ley Federal del Trabajo de 1931, la cual estipulaba la fijación de la jornada laboral, el descanso dominical, el salario mínimo, la reglamentación de las actividades de mujeres y niños, entre otros, así como la formación de sindicatos y las huelgas (OIT, 1936, p. 204). Un aspecto importante en el mejoramiento de las condiciones agrícolas era el conjunto de disposiciones que reglamentaban el riesgo profesional, como los accidentes y las indemnizaciones. El artículo 197

${ }^{14}$ El consejero técnico representante de los trabajadores chilenos, señor Godoy, señaló al respecto: "La importancia de la cuestión que me toca tratar es innegable; no se trata de sancionar convenios y de presentar a la consideración de los delegados la existencia de textos legales en los cuales se establezcan principios de protección a los obreros. Se trata, fundamentalmente, de denunciar la forma como ellos se aplican en relación con los sistemas de control y los organismos encargados de vigilar su cumplimiento y sancionar las evasiones a la ley" (oIT, 1936, p. 170).

\section{()(1) $(3$}


de la Ley Federal del Trabajo estipulaba una serie de obligaciones patronales con respecto a la situación de los trabajadores.

La delegación mexicana denunció que uno de los inconvenientes principales en la puesta en práctica de un sistema global de reglamentación de las actividades agrícolas era la ausencia de estadísticas sobre la materia. De esta forma, la CAT aprobó la resolución de recomendar al cA la realización de un estudio sobre el uso de las estadísticas en la agricultura, con el fin de avanzar en la elaboración de estadísticas uniformes (oIT, 1936, pp. 284-288).

Un cuarto tema abordado por la cAt fue el trabajo de mujeres y niños. La comisión respectiva reconoció el papel de la oIT en la promoción de las convenciones, apoyando las luchas históricas en favor de la maternidad y la protección de los menores, como forma de disminuir los altos índices de mortalidad infantil que golpeaba a la región. La igualdad entre hombres y mujeres, en lo que concierne a las remuneraciones y el acceso a los puestos de responsabilidad, fueron también planteados (oIT, 1936, pp. 267-268). Sobre el salario de las mujeres, el delegado gubernamental peruano Ramírez Otárola recomendó aprobar el ingreso mínimo en la industria a domicilio, actividad especialmente femenina, y que las mujeres solteras y casadas recibieran de manera íntegra su salario (OIT, 1936, p. 270). También propuso armonizar el seguro de maternidad en tres meses antes y tres meses después del parto, extendiendo sus beneficios a las actividades independientes y a la agricultura, aunque excluyendo la industria a domicilio y la pequeña producción agrícola. La comisión también avanzó en otro aspecto contenido en las convenciones internacionales, como era la obligación de los empresarios de instalar salas cunas en las empresas de más de 20 mujeres.

Algunos aspectos de la condición de las mujeres mostraron las diferentes posiciones existentes al interior de la comisión. La delegación chilena estuvo representada por María Ramírez, delegada del Movimiento Pro-Emancipación de las Mujeres de Chile, quien recomendó avanzar en medidas que favorecieran la igualdad de remuneraciones, cuestionando que fuera una conquista de los países de la región:

Tal como aparece en el Informe, muchos delegados han hablado de esta igualdad de salario como de una conquista obtenida gracias a las Convenciones redactadas en estas Conferencias [de Ginebra]. No creo que haya derecho moral de seguir hablando, por lo menos para los representantes de los países de la América del Sur, donde las mujeres están en condiciones que no son las de un ser humano. Aquí, delante de todos los Delegados a esta Conferencia,

\section{()(1)(3)}


tengo el deber de decir que esta conquista no existe ni ha existido jamás (oIT, 1936, p. 272).

Esta postura crítica de la delegada chilena se conectaba muy bien con las posiciones feministas clásicas, reflejando la importancia que tenía el trabajo en el movimiento feminista latinoamericano como factor de cambio social y reconocimiento de derechos. Según la historiadora Asunción Lavrin (2005), un aspecto que caracterizó al feminismo de la región a comienzos del siglo $\mathrm{xx}$ fue su objetivo de hacer compatible el trabajo con el hogar, así "la maternidad se convirtió en el hilo común que juntaba a la izquierda política, las feministas y los reformadores sociales liberales" (p. 20). Esto puede explicar el rechazo de la delegada chilena a las propuestas aprobadas por la comisión que limitaban el derecho al seguro de maternidad y que establecían el pago de $50 \%$ del salario a las mujeres embarazadas, proponiendo en cambio la creación de cajas de seguros de maternidad bajo la contribución patronal y el pago íntegro del sueldo, derechos que debían extenderse a la industria a domicilio y a la pequeña agricultura. Sin embargo, todas estas proposiciones fueron rechazadas por algunos delegados, especialmente peruanos y brasileños, bajo el argumento de que tales medidas aumentarían los costos de producción (oIT, 1936, p. 364).

En cuanto al trabajo infantil, la comisión expresó un solo desacuerdo: la fijación de la edad mínima. Aunque las delegaciones chilena y ecuatoriana propusieron la edad de 17 años y la de los Estados Unidos de 16, la cAT aprobó una resolución demandando la ratificación de la convención que fijaba en catorce años la edad mínima, especialmente en la industria (oIT, 1936, p. 370). De todas maneras la CAT recomendó al cA la revisión de las convenciones relativas al trabajo infantil con el fin de establecer la edad mínima en quince años, resolución que tuvo la oposición gubernamental de Brasil y patronal de Uruguay, Chile y Brasil (oIt, 1936, p. 371).

Por último, además de la ratificación de las convenciones, la condición de los trabajadores agrícolas y el trabajo infantil y de mujeres, la CAT aprobó resoluciones relativas a la alimentación popular y el salario mínimo. A propósito de lo primero, se apoyó la realización de una encuesta para determinar el estado de la nutrición y los costos de los alimentos, junto con la firma de tratados comerciales para procurar productos nutritivos a aquellos países que los necesitaran. La CAT aprobó también ajustar los salarios de los trabajadores al costo de la vida de sus respectivos países (oIt, 1936, pp. 289-290). En cuanto al salario mínimo, se resolvió solicitar al cA la incorporación, en

\section{()(1) $(3$}


el programa de una futura conferencia del trabajo, de la temática salarial y los subsidios familiares, según el criterio de satisfacción de las necesidades materiales y culturales (ort, 1936, p. 283).

\section{EL PROBLEMA SOCIAL INDÍGENA: LA ENCUESTA EN PERÚ DE 1936}

La mayor novedad de las resoluciones de la CAT fue colocar en el debate la realidad social de América Latina, muy distinta a la de Europa y Estados Unidos. Por ello, los delegados propusieron avanzar en el conocimiento de dos aspectos que estaban vinculados y que eran asumidos como los problemas más urgentes a tratar: la situación de la agricultura y las condiciones de vida de los indígenas.

La agricultura era un tema abierto a la intervención de la oit desde comienzos de los años de 1930, cuando se aprobó una serie de convenciones en ese ámbito, como por ejemplo la convención sobre el seguro de invalidez (1933), el seguro de muerte (1933) y el seguro de vejez (1933). Sin embargo, es necesario señalar que desde los años de 1920 la oit había establecido relaciones con el Instituto Internacional de Agricultura de Roma, creando en 1923 una comisión consultiva mixta agrícola cuyo objetivo era reglamentar la competencia entre las dos instituciones y avanzar en una colaboración más práctica. Pero la necesidad de una instancia fluida de cooperación, junto al reconocimiento de la problemática agrícola por parte de la institución de Ginebra, obligaron a formar una comisión permanente en 1938 (ort, 1938).

Fue en este marco que la CAT abordó el tema agrícola, resolviéndose solicitar al ca la realización de un estudio sobre las condiciones laborales en la agricultura y el nivel de desarrollo que tenían en el continente las estadísticas agrícolas (oIt, 1936, p. 391). La principal dificultad, que fue reconocida más tarde, era que la oIt no tenía un real conocimiento sobre estos aspectos porque su interés se había centrado en los sectores industriales y urbanos. Incluso, los países donde la agricultura era predominante no estaban en condición de entregar estadísticas detalladas y factibles de ser comparadas. Como se señaló en La Habana en 1939: "La primera tarea, entonces, consiste en reunir una información la más detallada posible sobre los diversos aspectos de las condiciones del trabajo agrícola en los diferentes países interesados" (оIт, 1939, p. 200). Luego del cierre de la CAT, las misiones realizadas por un funcionario del Servicio Agrícola de la oIT permitieron informar so-

\section{()(1) $(9$}


bre la situación de Argentina, Brasil y México, donde la agricultura era más representativa.

Al tema agrícola estaba asociada la cuestión indígena, discusión ausente de las conferencias internacionales, al menos hasta mediados de los años treinta. Los intelectuales habían comenzado a analizarla en los diversos congresos regionales, especialmente a partir del Primer Congreso de Previsión Social, llevado a cabo en Río de Janeiro en 1923, y el Primer Congreso de Economía Social de Buenos Aires en 1924. El Primer Congreso de Previsión Social señaló que la legislación laboral debía contener disposiciones especiales para los indígenas, garantizando su protección e incorporándolos a las actividades nacionales (oIT, 1928, p. 213).

La VII Conferencia Panamericana de 1933 en Montevideo solicitó a la Unión Panamericana la convocatoria a un congreso indigenista para 1939 en la ciudad de La Paz, encuentro que solamente tuvo lugar en 1940 en Pátzcuaro, México. El principal resultado de este congreso fue la creación de un Instituto Indigenista Interamericano, cuyo objetivo principal sería la promoción de una política de carácter continental (Confederación de Trabajadores de América Latina, 1940; Giraudo, 2006). Este instituto dispuso como órgano oficial de difusión su revista América Indígena, que comenzó a ser publicada desde 1941.

La primera dificultad para abordar la situación social indígena fue identificar sus alcances, producto de la ausencia de estadísticas precisas y porque el carácter racial de algunas ideologías progresistas no ayudaba a hacer visible su presencia, sobre todo en Argentina y Chile. ${ }^{15}$ La CAT aprobó la resolución presentada por el delegado gubernamental peruano Rebagliati relativa a las condiciones de vida y de trabajo de los indígenas del continente, resolviendo recomendar al ca la reunión de toda la información disponible sobre las dificultades que afectaban a dicho grupo, para luego realizar una encuesta sobre el tema como base de las futuras políticas sociales (oIt, 1936, pp. 147-148). La oIt, consciente de la amplitud del desafío, avanzó en la realización de estudios preliminares para luego emprender la realización de una investigación más completa en terreno. Estos estudios preliminares permitieron reunir información sobre algunos países -especialmente Boli-

${ }^{15}$ Esta apreciación, más bien técnica, está confirmada por los estudios del director del Instituto Indigenista Interamericano, Manuel Gamio, quien señala las dificultades de identificar correctamente a la población indígena y las complejidades de los censos que utilizaban el criterio lingüístico para determinar su número en un país. Véase Gamio (1942a y 1942b).

\section{()ㅜ(1) $\$$}


via, Ecuador y Colombia- aunque la encuesta profundizó sobre la condición indígena de uno solo: Perú. Según un informe posterior de 1939, la elección de este país formaba parte de una primera etapa:

La experiencia obtenida en la primera fase de la encuesta propuesta por la Conferencia de Santiago hizo que la ort llegase a la conclusión de que en adelante debería concentrar sus esfuerzos para lograr que todos los Gobiernos de los Estados americanos enviasen una detallada información sobre las condiciones de trabajo y de vida de sus poblaciones indígenas de manera que fuese posible hacer un estudio general comparativo de los problemas planteados (oIT, 1939, p. 175).

No es difícil encontrar las razones de la elección de Perú para la aplicación de la encuesta. La delegación peruana no sólo había demandado la aprobación de una resolución concreta sobre la materia, sino que también había puesto énfasis en la necesidad de acrecentar el conocimiento sobre el problema social indígena como base para la implementación de futuras acciones. A nivel más general, el segundo gobierno del general Óscar Benavides (1933-1939) se mostraba empeñado en mostrar logros en materia de políticas sociales y era favorable a los contactos con la oIT para recibir asesoría en diferentes temas (Drinot, 2016). Por último, es necesario agregar que la problemática indígena era un tema sensible en Perú, el cual podía mostrarse como un referente para el resto del continente. Sin ir más lejos las posiciones políticas pro indígenas habían ganado terreno en el país e intelectuales como José Carlos Mariátegui y Víctor Raúl Haya de la Torre, entre otros, centraban su discurso revolucionario en la defensa de su cultura, el mejoramiento de sus condiciones de vida y la reforma agraria (Devés, 2000). Por lo tanto, la OIT no podía quedar al margen de esta realidad, especialmente si se considera, como los mismos delegados a la CAT lo habían señalado, que la mayoría de la población trabajadora de los países de la costa del océano Pacífico era de origen indígena.

La encuesta en Perú fue llevada a cabo por el funcionario chileno de la oIT Moisés Poblete Troncoso, que había ingresado en 1927 luego de tener una importante carrera en Chile como director de la Oficina del Trabajo y subsecretario del Ministerio de Higiene, Asistencia, Trabajo y Previsión Social. Como funcionario internacional le había tocado realizar estudios sobre el avance de la legislación social en América Latina y misiones diplomáticas y de asistencia técnica en distintos países del continente (Yáñez, 2013). Por lo

\section{()(1) $(2$}


tanto, no sólo conocía muy bien el entramado de redes y vinculaciones que la oiт había establecido en la región, sino que también tenía muy buenas relaciones con intelectuales y funcionarios de primer nivel, siendo la persona más idónea para llevar a cabo la encuesta sobre la realidad indígena de Perú. Sin embargo, hubo también una razón práctica para su elección. La CAT de 1936 fue la instancia para plantear la necesidad de aumentar el número de funcionarios latinoamericanos en la OIT, así como el número de corresponsales en la región. ${ }^{16}$ Una de las corresponsalías fue creada en Santiago de Chile, siendo Moisés Poblete el responsable de organizarla. Todo indica que su traslado a América del Sur, acordado a comienzos de 1937, se debió al buen trabajo realizado en Perú y por la necesidad de "proseguir las encuestas sobre las condiciones de vida y de trabajo de la población indígena de ciertos países y para cumplir con otras tareas", como se señalaba en una carta confidencial de Poblete a Harold Butler. ${ }^{17}$

En febrero de 1936 el gobierno peruano dictó un decreto con el fin de facilitar las condiciones para que el comisionado de la oIt pudiera llevar a cabo su cometido, ofreciendo los medios técnicos y humanos del Ministerio del Trabajo (Poblete, 1938, p. 3) ${ }^{18}$ En marzo del mismo año se convocó a una sesión especial del Consejo Superior de Asuntos Indígenas donde expuso el asesor técnico José María Escalante sobre diversos problemas que afectaban a las comunidades locales. El director del Departamento del Trabajo, Edgardo Rebagliati, cooperó en la recolección de información y puso a disposición del delegado de la oit a Gerardo Bedolla Sáenz, uno de los especialistas del departamento, para que lo acompañara en sus viajes por las regiones mineras de Oroya y Morococha, situadas alrededor de $180 \mathrm{~km}$ al este de la capital.

Moisés Poblete participó en algunas conferencias científicas y se reunió con doce comisiones indígenas de distintas zonas de Perú, lo que le permitió tener un conocimiento directo de sus necesidades. Varios miembros del Congreso le ofrecieron documentación oficial sobre aspectos legislativos, además de acceder a las ideas de algunos intelectuales indigenistas. En este punto Poblete hace referencia a las obras de J. C. Tello, Hildebrando Castro Pozo, Abelardo Solís, José Carlos Mariátegui, Luis E. Valcárcel, Erasmo

${ }^{16}$ Luego de la CAT de 1936 fueron creadas oficinas de corresponsales en Santiago de Chile, La Habana, Caracas, Montevideo, Lima y Bogotá (oIt, 1939, p. 84).

${ }^{17}$ Carta confidencial de Poblete al director de la ort. M. Poblete Troncoso. Section administrative. Exp. P 2037, 1 de febrero de 1937. Archivo Organización Internacional del Trabajo (en adelante АоІт), Ginebra.

${ }^{18}$ El decreto se presenta en el anexo de este artículo.

\section{()(1) $\$$}


Roca, Atilio Sivirichi, Rafael Larco Herrera, entre otros: "Los indigenistas son numerosos en el Perú, ello revela un espíritu de alta comprensión de la importancia del problema, aunque no todos ellos se inspiran en los mismos principios ni preconizan iguales soluciones, seguramente por las dificultades y complejidad de las cuestiones" (Poblete, 1938, p. 5).

El informe de Poblete fue redactado desde fines de 1936 y durante gran parte de 1937, lo que explica su publicación en $1938 .{ }^{19}$ En términos formales comprende nueve capítulos, más una introducción, conclusión y anexos, con un total de 233 páginas, las que abordan aspectos históricos y las actividades de los indígenas en la agricultura, la industria y la minería. Además, analiza la presencia de algunas formas de trabajo obligatorio, problemas sociales específicos y los organismos existentes para su protección.

A partir de las estadísticas disponibles y de otras investigaciones, preparó un cuadro conteniendo el número y la distribución de las comunidades, lo que asociado a metodologías etnográficas y sociológicas le permitió establecer la existencia de un grupo primario que comprendía la población indígena propiamente tal y una gran cantidad de grupos secundarios, como mestizos, mestizo-japonés, mulatos, entre otros. Según Poblete (1938), se debía aplicar el concepto de "población indígena al grupo racial y étnico de la población peruana y al mestizo de indio, cuando sigue viviendo en las mismas condiciones económico-sociales que aquellos" (p. 13). De acuerdo con este criterio este grupo podía ser estimado en 800000 personas.

El informe hace referencia especialmente a la condición de los indígenas en la agricultura y la industria. En cuanto a lo primero, se describían las diversas modalidades de posesión de la tierra, desde la propiedad colectiva hasta la individual, estimando Poblete que la primera debía ser la base de las futuras cooperativas de producción, esenciales para la protección de la población. Los trabajadores agrícolas eran clasificados como inquilinos y jornaleros, dependientes del poder patronal y con condiciones laborales precarias, existiendo todavía modalidades de trabajo obligatorio. En lo que respecta a las actividades industriales, el informe daba cuenta de la presencia indígena en las manufacturas, principalmente urbanas y mineras. Aunque el artesanado no se veía afectado por el desarrollo de la industria o por la presencia

19 De acuerdo con el archivo personal de Poblete, el 24 de junio de 1936 solicitó un periodo de reposo para tratar "la enfermedad al corazón que tuve durante mi misión en América del Sur”. M. Poblete Troncoso. Section administrative. Exp. P 2037, 24 de junio de 1936. AOIT, Ginebra.

\section{()(1) $\$$}


de productos extranjeros, se especulaba que las manufacturas tradicionales, como los textiles y la alfarería, desaparecían por la reducción de la población indígena o por su aislamiento geográfico. Ante la ausencia de legislación especial para los trabajadores industriales de origen indígena, Poblete proponía reforzar las medidas de control de algunas leyes, en particular sobre la jornada laboral, los accidentes y el sistema de enganche. Se planteaba también la necesidad de limitar algunas formas de trabajo obligatorio, como el transporte de mercancías y el trabajo forzado y gratuito en las obras públicas. El informe proponía también mejorar las condiciones de vida de los indígenas que residían en las ciudades, a partir del mejoramiento de sus viviendas, aunque se reconocía el progreso en la construcción de barrios obreros.

En una arista más sanitaria, Poblete señaló que los problemas más complejos que afectaban a la población indígena peruana eran el alcoholismo y el consumo de coca. Si bien reconocía la existencia de un marco cultural que explicaba dichas conductas, ponía el acento en el papel de la educación y de la ley para hacerles frente, porque de una manera disimulada podían afectar profundamente su desarrollo como grupo.

Por último, el informe señalaba que uno de los mayores desafíos que enfrentaba Perú era la integración de su población indígena, de tal forma de hacerla partícipe de la vida social, económica y cultural del país y "de hacerla capaz de tomar parte activa en la producción y en el consumo y de ejercer sus derechos ciudadanos con la misma conciencia y capacidad que el resto de la población" (Poblete, 1938, p. 183). Esto traería consecuencias beneficiosas no tan sólo para los indígenas sino también para el desarrollo económico y social del país:

Es también necesario proteger el capital humano que el indígena representa, elevar progresivamente su standard de vida para permitirle gozar de las conquistas de la civilización y del progreso. La valorización de este capital humano debe realizarse en atención al número de indígenas y a los factores especiales del país. Él es fuente de riqueza y de progreso general, que de ninguna manera debe abandonarse (Poblete, 1938, p. 197).

La importancia del estudio de Poblete radica en que ayudó a hacer visible para la oIt el problema social indígena, ampliando el conocimiento en cuanto a sus dimensiones y alcances. Sin embargo, y pese a estos avances, no es sino hasta los años de 1940 que se establece un balance más completo sobre la situación indígena del continente. La principal dificultad seguía

\section{()(1) $(9$}


siendo la falta de estadísticas confiables para dimensionar el problema, ante lo cual el director de la oIt, Edward Phelan, señaló en un informe de 1946 lo siguiente:

No está de más el hacer notar aquí que es muy difícil, desde todo punto de vista, el establecer la calidad de "mestizo" o la calidad de "blanco", así como el llegar a obtener características específicas que justifiquen la denominación de "indio". Muchos investigadores han empleado para clasificar a los indios la pigmentación cutánea $\mathrm{u}$ otros rasgos somáticos que se han revelado de poca importancia, existiendo la tendencia cada vez más acentuada de tomar como punto de diferenciación los caracteres culturales y económicos entre los distintos grupos de la población (orT, 1946, pp. 129-130).

El informe del director de la oIT incorporó como aspecto nuevo la seguridad social, ausente en el estudio de Poblete, planteando la ayuda médica "como un primer paso hacia la aplicación de regímenes de seguridad social" (oIT, 1946, p. 133). Además, se señalaba que toda puesta en práctica de una política social indígena debía darse en el marco de la cooperación interamericana y con el apoyo de la OIT, "la única que puede permitir el aprovechamiento de las experiencias realizadas en este o en otros continentes y que sin duda podrá llegar a obtener las soluciones más justas que conduzcan a la integración total del indio en la vida económica de los pueblos de América" (oIT, 1946, p. 141).

En el curso de los años de 1940, la región podía mostrar ciertos progresos en materia legislativa y de defensoría indígena, tal como se presentan en el cuadro 1.

Esta política continental puede ser caracterizada como parte de una visión paternalista de la acción del Estado, orientada a la protección de la "raza indígena" y su integración a la vida nacional, justificando para ello la creación de servicios encargados de resolver sus problemas y, más concretamente, del cargo de procurador, tal como lo solicitó el Primer Congreso Indigenista de $1940 .^{20}$

La Tercera Conferencia Americana del Trabajo, de 1946, realizada en la ciudad de México, incapaz de llegar a una resolución compartida por el conjunto de los delegados, solicitó al cA que prosiguiera con sus estudios e informes, con la esperanza de abordar la problemática indígena en las próxi-

${ }^{20}$ Véanse las resoluciones en Oficina de Asuntos Indígenas (1948, pp. 22-23).

\section{(ㄷ)(1) $(3$}




\section{Cuadro 1. Legislación Social Indígena}

Bolivia Decreto de 1945 Prohibición del trabajo forzado de la población indígena.

Brasil Decretos de 1942 Servicio de protección de los indígenas, estableciy 1945

Colombia Ley de 1943 do en el Ministerio de Agricultura. Colonias agrícolas y ganaderos se establecen para la protección de la población indígena.

Chile $\quad$ Ley de 1944

Las tierras de las comunidades indígenas son declaradas libres de impuestos.

Ecuador Decreto de 1943 Creación del Departamento de Servicios de Asuntos Indígenas.

México Decreto de 1941 Creación del Departamento de Asuntos Indígenas.

Perú Decreto de 1941 Creación del puesto de procurador indígena, dependiente del Ministerio del Trabajo.

Fuente: OIT (1946, pp. 134-137).

mas conferencias, tal como se hizo en la cuarta conferencia de 1949, en Montevideo (oIT, 1949). Todo este programa de protección concluyó, en una primera etapa, con la aprobación de la primera convención internacional sobre pueblos indígenas y tribales en $1957 .^{21}$

\section{CONCLUSIONES}

Durante los años 1930 y 1940 el continente americano se presentó como un espacio mayor en la escala de las relaciones internacionales, apareciendo modelada por disputas globales donde participaban la SDN, el panamericanismo y otras instituciones satélites del periodo de entreguerra. En particular, América Latina tenía un conjunto de problemas y demandas que no habían sido incorporados en la creación de la oIT en 1919, la que había privilegia-

${ }^{21}$ La convención 107 de 1957 incluye aspectos como el derecho a la tierra, contratación y condiciones laborales, formación profesional, seguridad social, salud, educación y medios de comunicación. Además, aborda materias territoriales y de recursos, los que serían la base de la convención 169.

\section{()ㅜ(1) $\$$}


do abordar los aspectos más tradicionales del mundo del trabajo y de la industria.

Los efectos de la Gran Depresión hicieron visibles problemas nuevos, que daban cuenta de mejor forma de las particularidades del continente y de sus aportes al debate de las instituciones internacionales como la oIt. Fue en este contexto que se desarrollaron las conferencias interamericanas del trabajo, las que facilitaron la reunión de expertos, la discusión con un enfoque regional de los problemas continentales y la realización de estudios y de misiones técnicas.

La importancia asignada en este artículo a la cAT llevada a cabo en Santiago de Chile en enero de 1936 se explica justamente porque los debates se centraron en aquellas propuestas sugeridas por las mismas delegaciones del continente y que no tenían espacios en las conferencias internacionales de la oIт. Pero, además, la cAT tuvo consecuencias más prácticas en el internacionalismo continental, como fue el desplazamiento de expertos y de misiones técnicas, lo que suponía poner en práctica una política social a nivel regional. Aunque muchas de esas misiones estuvieron orientadas hacia un solo país, tenían el interés de organizar un cuadro más global, sobre todo en aquellos problemas concernientes a varias naciones, donde la situación de los indígenas y de la agricultura son los mejores ejemplos. El impacto de las encuestas y misiones de asistencia técnica de los años 1930 y 1940, y donde la encuesta en Perú fue una de entre muchas otras, fue importante para el desarrollo de las competencias de la orT. Su interés en conocer la dimensión de los problemas sociales del continente es la prueba de la apertura a establecer una relación más directa con los países de la región.

Los principales aportes de la encuesta realizada por Moisés Poblete pueden ser sintetizados en dos aspectos. Primero, siendo una de las primeras misiones que se llevaron a cabo en América Latina por parte de la OIT, ayudó a formalizar sus protocolos de intervención, reforzando los vínculos con las autoridades locales y legitimando las relaciones de cooperación con la institución de Ginebra. Segundo, ayudó a conocer los alcances del problema social indígena, sirviendo de orientación a futuros estudios sobre la materia y permitiendo establecer comparaciones con las realidades de otros países. Pero quizá lo más relevante es que puede ser considerado el primer paso en los sucesivos aportes que realizará la oIT para diagnosticar y proponer mejoras en las condiciones sociales de la población indígena, política protectora que concluirá en una primera etapa con la aprobación de la convención 107 de 1957.

\section{()(1) $(3$}


¿Cuánto de las conclusiones ofrecidas por Poblete sobre la realidad indígena sirvieron para futuros estudios sobre la temática? ¿Cómo fue percibido este trabajo en los esfuerzos que realizaba la oit para comprender las particularidades de la región? ¿Cuáles fueron las vías de difusión que tuvo el informe? En este sentido, no deja de ser paradójico el poco impacto que tuvo este primer esfuerzo por conocer la realidad indígena del continente en el entramado administrativo de la institución de Ginebra, tal como ha sido destacado por diversos autores (Rodríguez-Piñero, 2005), pese a los propios empeños que realizó Poblete por difundir su investigación en algunas instancias de reunión continental. Por ejemplo, se sabe que participó en la VII Conferencia Panamericana de Lima en 1938, donde se abordó, entre otros puntos, la "consideración del estado de las poblaciones indias", oportunidad que le permitió entregar una copia de la publicación al líder sindical mexicano, y presidente de la CTAL, Vicente Lombardo Toledano. ${ }^{22}$

Más allá de estos esfuerzos de difusión del informe de Poblete, creemos que existieron al menos dos condiciones que hicieron que esta primera tarea de conocer la cuestión indígena no tuviera el reconocimiento posterior. En primer lugar, en septiembre de 1939 se inició la segunda guerra mundial, que tuvo para la oIт y su trabajo de internacionalización un efecto crítico y en un primer momento paralizante, teniendo que trasladar sus operaciones a Canadá. La guerra no sólo fue contraproducente en el plano de las comunicaciones cotidianas, sino también en sus efectos sociales y económicos, movilizando los esfuerzos de los gobiernos hacia otros intereses, redundando en una disminución de la participación de los países en las conferencias internacionales y una reducción en el número de ratificaciones. En concreto, nos parece que la continuidad de estos primeros esfuerzos por hacer visibles las particularidades regionales se vio interrumpida hasta entrados los años de 1940, cuando, al menos formalmente, se convocó a la Tercera Conferencia Interamericana del Trabajo, en México (1946). ${ }^{23}$ En segundo lugar, el acercamiento a la problemática indígena se hizo durante los años de 1930 desde un

${ }^{22}$ Para el historiador Patricio Herrera, especialista en la obra de Vicente Lombardo Toledano y la стAL, "La investigación de Poblete fue importante para que la CтAL acentuara su posición al respecto [la situación indígena] en la Segunda Conferencia 'Americana' del Trabajo, celebrada en La Habana en 1939, la cual puede ser considerada como el último antecedente para la realización de Primer Congreso Indigenista Interamericano" (Herrera, 2013a, p. 152).

${ }^{23}$ El informe de la ort de 1953 sobre las poblaciones indígenas señala a propósito del estudio de Poblete: "La segunda guerra mundial impidió la extensión de este tipo de encuestas a otros países del continente" (OIT, 1953, p. 624).

\section{()(1) $\$$}


plano más bien cultural y étnico, dejando de lado su dimensión sociolaboral. Las modalidades precapitalistas de producción, sumadas a formas de trabajo colectivas y obligatorias, dificultaron la construcción de un imaginario del indígena como trabajador $y$, en consecuencia, su inserción en el universo conceptual de la огт. Harold Butler, en un informe de 1946, señaló al respecto:

Como se ve, la mayor parte de las resoluciones señaladas tratan el problema indígena desde un punto de vista ya sea cultural, etnológico, filológico o se refieren muy de paso a los problemas derivados de la aplicación de la legislación social y del trabajo entre estos trabajadores. Sin embargo, y aparte de lo acordado a este respecto en Pátzcuaro, la Confederación de Trabajadores de América Latina (стAL) y las Conferencias del Trabajo de los Estados de América miembros de la Organización Internacional del Trabajo, han aprobado algunas declaraciones concretas sobre el indio en su carácter de trabajador (ort, 1946, p. 139).

En cierta forma la encuesta de Poblete formaba parte de este grupo de estudios que habían colocado demasiado énfasis en conocer la dimensión cultural de los indígenas, sin abordar en profundidad sus condiciones laborales y regulaciones legislativas, aspectos esenciales de la misión de la ort.

\section{ANEXO DOCUMENTAL}

Gobierno del Perú.

Ministerio del Trabajo

Decreto del 29 de febrero de 1936.

Considerando:

Que en la reciente Conferencia del Trabajo de los Estados de América se aprobó, a propuesta de la delegación gubernamental peruana y de acuerdo con las instrucciones que le fueron impartidas, una resolución orientada a solicitar de los gobiernos del Continente, interesados en la solución de los problemas económicos y sociales de la vida y trabajo de las poblaciones indígenas, el envío a la Oficina Internacional del Trabajo de los antecedentes y documentos que guarden relación con ellos, y que, además, verifique la misma Oficina Internacional del Trabajo un estudio especial de esos problemas y que se tomen en cuenta las posibilidades que existen para llegar a una acción internacional determinada con un objeto práctico;

\section{()(1) $\$$}


Que, en observancia de ese acuerdo, la Oficina Internacional del Trabajo ha designado al señor don Moisés Poblete Troncoso, funcionario Titular Americano de dicha Organización, como comisionado especial para que, constituyéndose en nuestro país y en otros del Continente americano, realice los estudios pertinentes;

Que, tratándose de una iniciativa que se refiere a la cuestión social que constituye preocupación preferente del Gobierno y en la que toda cooperación es por lo mismo apreciable, y más todavía cuando, como en el presente caso, ella tiene la autoridad técnica de la Oficina Internacional del Trabajo

Se resuelve:

1. La Dirección del Trabajo, Previsión Social y Asuntos Indígenas, queda encargada:

a) De prestar atención y facilidades al Delegado Especial de la Oficina Internacional del Trabajo, adscribiéndole, además, un asesor especial que sea miembro de la Sección de Asuntos Indígenas.

b) De efectuar el trabajo a que se contrae la primera parte de la resolución adoptada por la Conferencia del Trabajo de los Estados americanos, y de reunir y coordinar en consecuencia, la documentación y las observaciones relacionadas con los problemas económicos y sociales de vida y trabajo de nuestra población aborigen.

2. Las autoridades políticas de la República prestarán al comisionado de la Oficina Internacional del Trabajo las facilidades que requiera para el mejor cumplimiento de su cometido.

Regístrese y comuníquese.

Firma del Presidente de La República.

Firma del Ministro del Trabajo.

\section{LISTA DE REFERENCIAS}

Aglan, A., Feiertag, O. y Kévonian, D. (eds.) (2011). Humaniser le travail. Régimes économiques, régimes politiques et Organisation Internationale du Travail (1929-1969). Bruselas: Peter Lang.

Caruso, L. (2016). Embarcados, los trabajadores marítimos y la vida a bordo: Sindicato, empresas y Estado en el puerto de Buenos Aires, 1889-1921. Buenos Aires: Imago-Mundi.

Confederación de Trabajadores de América Latina (1940). Primer Congreso Indigenista Interamericano. México: Autor.

Devés, E. (2000). Del Ariel de Rodó a la CEPAL (1900-1950). Buenos Aires: Editorial Biblos-Dibam. 
Drinot, P. (2016). La seducción de la clase obrera. Trabajadores, raza y la formación del Estado peruano. Lima: Instituto de Estudios Peruanos.

Drinot, P. y Knight, A. (2015). La Gran Depresión en América Latina. México: FCE.

Dumont, J. (2008). L'Institut International de Coopération Intellectuelle et le Brésil (19241946). Le pari de la diplomatie culturelle. París: IHEAL.

Gallardo, G. (1941). Panamericanismo. Santiago: Editorial Nascimiento.

Gamio, M. (abril, 1942a). Consideraciones sobre el problema Indígena en América. América Indígena, II (2), 17-23

Gamio, M. (julio, 1942b). Las características culturales y los censos indígenas. América Indígena, II(3), 15-19.

García Oldini, F. (24 de junio, 1935). Discussion du rapport du Directeur. Informations Sociales. 13.

Giraudo, L. (julio-septiembre, 2006). El Instituto Indigenista Interamericano y la participación indígena (1940-1998). América Indígena, 3, 6-34.

González, J. (2000). El reconocimiento del derecho indígena en el convenio 169 de la orT. En J. E. Ordóñez (ed.), Análisis interdisciplinario del Convenio 169 de la oIT: IX Jornadas Lascasianas. México: IIJ-UNAM.

Herrera, P. (2013a). La Confederación de Trabajadores de América Latina y la implementación de su proyecto sindical continental (1938-1941). Trashumante, 2, 136-164.

Herrera, P. (2013b). La primera conferencia regional del trabajo en América: su influencia en el movimiento obrero, 1936. En F. Herrera y P. Herrera (eds.), Redes, cooperación técnica e institucionalidad social, 1919-1950 (pp. 199-242). Morelia: IIH-Universidad Michoacana de San Nicolás de Hidalgo.

Kelchner, W. (1931). Fundación y desarrollo de la Unión Panamericana. Washington: Unión Panamericana.

Kott, S. y Droux, J. (eds.) (2012). Globalizing social rights: The International Labor Organization and beyond. Nueva York: Palgrave.

Lavrin, A. (2005). Mujeres, feminismo y cambio social en Argentina, Chile y Uruguay. 1890-1940. Santiago: Dibam.

Lespinet-Moret, I. y Viet, V. (eds.) (2011). L'Organisation Internationale du Travail. Origine, développement, avenir. Rennes: Presses Universitaires de Rennes.

Lobato, M. (enero-noviembre, 2007). Historia de las instituciones laborales en Argentina: una asignatura pendiente. Revista del Trabajo, 4, 145-154.

Manger, W. (1931). The Pan American Union and the Pan American Conferences. Washington: Pan American Union.

Marichal, C. (ed.) (2002). México y las Conferencias Panamericanas. México: Secretaría de Relaciones Exteriores.

\section{()(1) $\$$}


Ministerio de Industria y Trabajo (1937). Texto de las resoluciones adoptadas por la Conferencia del Trabajo de los Estados de América, miembros de la Organización Internacional del Trabajo, celebrada en Santiago de Chile en enero de 1936. Montevideo: Imprenta Nacional.

Naón, R. (1919). La guerra europea y el panamericanismo. Nueva York: Asociación Americana para la Conciliación Internacional, División Interamericana.

Oficina de Asuntos Indígenas (1948). Acta final del Primer Congreso Indigenista Interamericano. México: Instituto Indigenista Interamericano.

Organización Internacional del Trabajo (1928). Legislación social de América Latina. Vol. I. Ginebra: Autor.

Organización Internacional del Trabajo (1936). Conferencia del Trabajo de los Estados de América miembros de la Organización Internacional del Trabajo. Ginebra: Autor.

Organización Internacional del Trabajo (1938). Les problèmes sociaux dans l'agriculture. La commission permanente agricole du Bureau International du Travail. Ginebra: Autor.

Organización Internacional del Trabajo (1939). Segunda Conferencia del Trabajo. Informe acerca de las medidas tomadas para dar cumplimiento a las resoluciones adoptadas por la Conferencia de Santiago de Chile. Ginebra: Autor.

Organización Internacional del Trabajo (1946). Tercera Conferencia del Trabajo. Memoria del Director. Montreal: Autor.

Organización Internacional del Trabajo (1949). Cuarta Conferencia de los Estados de América miembros de la огт. Condiciones de vida y de trabajo de las poblaciones indígenas de América Latina. Ginebra: Autor.

Organización Internacional del Trabajo (1953). Poblaciones indígenas. Condiciones de vida y de trabajo de los pueblos autóctonos de los países independientes. Ginebra: Autor.

Pernet, C. (2011). L'ort et la question de l'alimentation en Amérique Latine (19301950). En Lespinet-Moret y Viet (dir.), L'Organisation Internationale du Travail. Origine, développement, avenir (pp. 167-192). Rennes: Presses Universitaires de Rennes.

Plata, V. (2013). La difusión de las normas internacionales del trabajo en Venezuela, 1936-1939: Una práctica de cooperación técnica internacional en la orT. En F. Herrera y P. Herrera (eds.), Redes, cooperación técnica e institucionalidad social, 1919-1950 (pp. 127-160). Morelia: IıH-Universidad Michoacana de San Nicolás de Hidalgo.

Poblete, M. (1938). Condiciones de vida y de trabajo de la población indígena del Perú. Ginebra: OIT.

Quesada, E. (1916). El nuevo panamericanismo y el congreso científico de Washington. Buenos Aires: Talleres Gráficos del Ministerio de Agricultura de la Nación.

Quesada, E. (1919). La evolución del panamericanismo. Buenos Aires: Talleres Gráficos del Ministerio de Agricultura de la Nación.

\section{(ㅇ)(1) $(3$}


Rodríguez-Piñero, L. (2005). Indigenous peoples, postcolonialism, and international law. The ILO regime (1919-1989). Oxford: Oxford University Press.

Sheinin, D. (ed.) (2000). Beyond the ideal: Pan Americanism in Inter-American Affairs. Westport-Londres: Praeger.

Soprano, G. (2007). Del Estado en singular al Estado en plural. Contribución para una historia social de las agencias estatales en la Argentina. Cuestiones de Sociología, 4, 19-48.

Van Daele, J., Rodríguez, M., Van Goethem, G. y Linden, M. (eds.) (2010). ILo histories: Essays on the International Labour Organization and its impact on the world during the twentieth century. Berne: Peter Lang.

Wehrli, Y. (2003). Créer et maintenir l'intérêt: la liaison entre le Secrétariat de la SDN et l'Amérique Latine (1919-1929) (Tesis de licenciatura inédita). Universidad de Ginebra, Suiza.

Yáñez, J. C. (2008). La intervención social en Chile (1907-1932). Santiago: Ril Editores, 2008.

Yáñez, J. C. (2013). La oit y la red sudamericana de corresponsales. El caso de Moisés Poblete, 1922-1946. En F. Herrera y P. Herrera (ed.). Redes, cooperación técnica e institucionalidad social, 1919-1950 (pp. 25-61). Morelia: IIH-Universidad Michoacana de San Nicolás de Hidalgo.

Yáñez, J. C. (2014). L'ort et l'Amérique du Sud (1919-1949). La construction d'un laboratoire social régional (Tesis de doctorado inédita). eHess-París, Francia.

\section{OTRAS FUENTES}

Archivos

AN Archivo Nacional, Chile.

AOIT Archivo Organización Internacional del Trabajo, Ginebra. 